\title{
Interação de ondas da superfície do mar com obstáculos flutuantes e submersos
}

\author{
Rômulo Lima da Gama* \\ Instituto de Matemática, PPGMAp, UFRGS, \\ 91509-900, Porto Alegre, RS \\ E-mail: lima.gama@ufrgs.br, \\ Leandro Farina \\ Universidade Federal do Rio Grande do Sul- Instituto de Matemática \\ 91509-900, Agronomia, Porto Alegre, RS \\ E-mail: farina@mat.ufrgs.br
}

\section{RESUMO}

A interação das ondas oceânicas com corpos flutuantes e submersos é um problema constantemente estudado e presente na literatura, como em [1]. Os principais casos estudados são na presença de uma superfície livre limitando o fluido. Em [3] é apresentado o caso conhecido como problema de doca, onde o obstáculo flutua. A solução deste problema pode ser obtida resolvendo a equação integral de contorno para um potencial de velocidade $\phi$. O segundo caso, onde o corpo está completamente submerso, é investigado em [2]. A principal característica notada é a ocorrência de frequências resonantes onde a força hidrodinâmica assume máximos locais.

Neste trabalho iremos estudar as ondas e suas implicações em estruturas rígidas com componentes finos submersos, que são partes integrantes de plataformas construídas para atuarem na indústria do petróleo. Inicialmente vamos considerar como obstáculo um disco plano. Matematicamente esse problema permite uma formulação em termos de uma equação integral cuja solução pode ser obtida expandindo o potencial de velocidade em funções de Legendre.

Supomos que os movimentos do fluido são de pequena amplitude, simplesmente harmônicos e que o mesmo é incompressível e não viscoso. Denotamos por $\phi$ o potencial de velocidade e por $[\phi]$ a descontinuidade em $\phi$ sobre a superfície do disco. As condições satisfeitas por $\phi$, são a equação de Laplace,

$$
\left(\frac{\partial^{2}}{\partial x^{2}}+\frac{\partial^{2}}{\partial y^{2}}+\frac{\partial^{2}}{\partial z^{2}}\right) \phi=0, \text { no fluido, }
$$

juntamente com a condição da superfície livre

$$
K \phi+\frac{\partial \phi}{\partial z}=0 \mathrm{em} z=0
$$

onde $K=\frac{\omega^{2}}{g}$. Na superfície do corpo, a velocidade normal é prescrita por $\frac{\partial \phi}{\partial n}=V$, onde $V$ é uma função conhecida e $\frac{\partial \phi}{\partial n}$ denota a derivada normal.

Adicionalmente, $\phi$ satisfaz uma condição de radiação:

$$
r^{1 / 2}\left(\frac{\partial \phi}{\partial r}-i K \phi\right) \rightarrow 0 \text { quando } r=\left(x^{2}+y^{2}\right)^{1 / 2} \rightarrow \infty .
$$

A função de Green para este problema é dada por

$$
G(P, Q) \equiv G(\xi, \eta, \zeta ; x, y, z)=G_{0}(R, z-\zeta)+G_{1}(R, z+\zeta),
$$

\footnotetext{
* Mestrando em Matemática Aplicada/PRH-216
} 
onde $R=\left((x-\xi)^{2}+(y-\eta)^{2}\right)^{1 / 2}, G_{0}=\left(R^{2}+(z-\zeta)\right)^{-1 / 2} \mathrm{e}$

$$
G_{1}=\int_{0}^{\infty} e^{-k(z+\zeta)} J_{0}(k R) \frac{k+K}{k-K} d k .
$$

Aqui $J_{0}$ é a função de Bessel de ordem zero.

Para qualquer função harmônica, satisfazendo $\phi=O\left(r^{-1}\right)$ quando $r \rightarrow \infty$, temos a partir da segunda identidade de Green, a seguinte representação

$$
\phi(P)=\frac{1}{4 \pi} \int_{S}\left(\phi(q) \frac{\partial}{\partial n} G(P, q)-G(P, q) \frac{\partial \phi}{\partial n}\right) d S .
$$

Agora, para um corpo fino submerso com superfície $\Omega$, definimos a descontinuidade em $\phi$ por $[\phi]=\lim _{Q \rightarrow q^{+}} \phi(Q)-\lim _{Q \rightarrow q^{-}} \phi(Q)$, onde $q$ denota um ponto na superfície do corpo e $Q$ um ponto no fluido.

Aplicando a condição da velocidade normal na superfície do corpo, temos

$$
V(p)=\frac{1}{4 \pi} \oint_{\Omega}[\phi(q)] \frac{\partial^{2}}{\partial n_{q} \partial n_{p}} G(p, q) S_{q} .
$$

A integral anterior pode ser interpretada como de parte-finita de Hadamard.

Seja $\Omega: z=F(x, y)+\frac{b}{2} \in D$, onde $D$ é o disco unitário e $\frac{b}{2}$ é a profundidade que o corpo está submerso. Considerando algumas notações e definições, como $\omega(x, y)=[\phi(q)]$, pode-se reescrever a equação (7) como

$$
V(p)=\frac{1}{4 \pi} \oint_{D} H \omega(x, y) d x d y+\frac{1}{4 \pi} \int_{D} W \omega(x, y) d x d y,(x, y) \in D,
$$

onde $W$ e $H$ são os núcleos da equação integral. $H$ possui uma hipersingularidade em $x=y$.

A Equação (8) é a equação integral hipersingular governante para o problema de um disco circular não plano submerso na água com profundidade infinita. Sua solução dá o salto no potencial velocidade $[\phi]$ sobre $\Omega$.

Nosso principal objetivo é desenvolver um algoritmo para o cálculo numérico de funções de Green para obstáculos finos submersos e não planos, tais como superfícies rugosas. Consideraremos o núcleo da equação integral (8) exato, ou seja, a superfície não-plana do obstáculo não é aproximada na forma $F(x, y)=\epsilon f(x, y)$, como no trabalho de Ziebell e Farina [4], onde um método pertubativo é empregado e soluções de ordem $\epsilon$ são obtidas numericamente.

Para avaliar numericamente a função de Green nos baseamos no algoritmo original de Newman [5], o qual reescreve $G_{1}$ em termos de funções de Bessel e de Struve, assim evitando o cálculo da integral de contorno singular.

As funções de Green são importantes para descrever o escoamento do fluido e as ondas, consequentemente as forças devidas a elas em tais obstáculos. Essas forças podem ser expressas através da massa adicional e do coeficiente de amortecimento.

Palavras-chave: Ondas Oceânicas, Equações integrais, Hadamard

\section{Referências}

[1] H. Lamb , "Hydrodynamics", Cambridge University Press. Sexta edição, (1932).

[2] P. A. Martin; L. Farina, Radiation of water by a heaving submerged horizontal disc, J. Fluid Mech.,337, 365-379, 1997

[3] J. W. Miles, On surface-wave forcing by a circular disc,J. Fluid. Mech., 175,97-108,1987.

[4] J. S. Ziebell; L. Farina, Water wave radiation by a submerged rough disc, Wave Motion 49, 3449,2012 .

[5] J. N. Newman, Algorithms for the free-surface Green function, J. Eng. Math., 19, 57-67, 1985. 